\title{
Níveis de qualidade de vida em idosas ativas praticantes de dança, musculação e meditação
}

The levels of quality of life among active elderly women practitioners of dance, strengthining and meditation

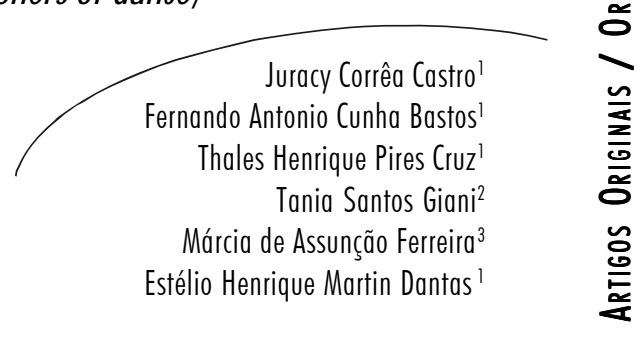

\section{Resumo}

O objetivo deste estudo foi comparar os níveis de qualidade de vida entre idosas sedentárias e ativas, praticantes de dança, musculação e meditação, avaliando ainda os efeitos destes sobre aqueles. Para tal, neste estudo ex post facto, a amostra foi dividida em quatro grupos: grupo de dança (GD, $\mathrm{n}=20$, idade $=67 \pm 4$ anos), grupo de musculação (GF, $n=15$, idade $=67 \pm 5$ anos), grupo de meditação (GM, $\mathrm{n}=15$, idade $=68 \pm 4$ anos) e grupo controle (GC, $\mathrm{n}=20$, idade $=68 \pm 6$ anos). $O$ protocolo utilizado na avaliação da qualidade de vida foi o questionário World Health Organization Quality of Life Group-old WHOQOL-OLD. Nas comparações múltiplas (Post Hoc de Sheffe ou intervalo de confiança) foram encontrados resultados satisfatórios nas variáveis dom1 (Habilidades sensoriais; GDxGC, $\mathrm{p}=0,006$ ), dom2 (Autonomia; GDxGF, $p=0,026$; GDxGC, $p<0,001$; GFxGC, $p=0,001$; GMxGC, $p<0,001$ ), dom3 (Atividades passadas presentes e futuras; GDxGC, $p=0,004$; GFxGC, $\mathrm{p}=0,033 ; \mathrm{GMxGC}, \mathrm{p}<0,001$ ), dom4 (Participação social; GDxGC, $\mathrm{IC}=+0,421,+41,479$; $\mathrm{GMxGC}, \mathrm{IC}=+4,456,+48,804)$ e $\mathrm{QVG}(\mathrm{GDxGC}$,

\section{Palavras-chave:} Idoso. Mulheres. Saúde da Mulher. Exercícios de Alongamento Muscular. Estudo Comparativo. Qualidade de Vida. Musculação. Meditação. Dança.

Universidade Castelo Branco, Pós-Graduação em Ciência da Motricidade Humana, Laboratório de Biociências da Motricidade Humana. Rio de Janeiro, RJ, Brasil.

2 Universidade Estácio de Sá, Centro de Ciências Biológicas e da Saúde, Farmácia e Fisioterapia. Rio de Janeiro, RJ, Brasil

3 Universidade do Estado do Rio de Janeiro, Pós-Graduação e Pesquisa da Escola de Enfermagem Anna Nery

Correspondência / Correspondence

Juracy Correa Castro

Centro Federal de Educação Tecnológica do Pará

Coordenação de Educação Física

Av. Almirante Barroso, 1155

66093-020 - Belém, Pará, Brasil

E-mail: jura_teclado@yahoo.com.br 
$\mathrm{p}<0,001$; GFxGC, $\mathrm{p}=0,033$; GMxGC, $\mathrm{p}<0,001)$. Após a exposição dos dados, inferiu-se que os dois programas de atividade física (dança e musculação) e a prática da meditação apresentaram, na amostra analisada, resultados satisfatórios, contribuindo para um melhor nível de qualidade de vida quando comparados com aqueles apresentados pelo GC.

\section{Abstract}

This study aimed to compare the levels of quality of life between active and sedentary elderly, practitioners of dance, meditation and strengthening. So in this study ex post facto, the sample was divided into four groups: dance group (DG, $\mathrm{n}=20$, age $=67 \pm 4$ years), strength group (SG, $\mathrm{n}=15$, age $=67$ \pm 5 years), meditation group ( $M G, n=15$, age $=68 \pm 4$ years) and the control group (CG, $\mathrm{n}=20$, age $=68 \pm 6$ years). The protocol used to assess the quality of life was the questionnaire World Health Organization Quality of Life Group-old - WHOQOL-OLD. In multiple comparisons (Post Hoc Sheffe, or confidence interval) we found satisfactory results in the variables dom1 (Sensory abilities; DGxGC, p = 0006), dom2 (Autonomy; DGxSG, p = 0026; DGxGC, p <0001; SGxGC, p = 0001; MGxGC, p <0001), dom3 (past present and future activities; DGxGC, p = 0004; SGxGC, p = 0033; MGxGC, p <0001), dom4 (Social Participation; DGxGC, CI $=+0421$, +41479; MGxGC, CI = + 4456, 48804), and QVG (DGxGC, p <0001; SGxGC, $\mathrm{p}=0033 ; \mathrm{MGxGC}, \mathrm{p}<0001)$. After data exposure, we inferred that the two programs of physical activity (dance and strenghtining) and the practice of meditation had satisfactory results in the trial, contributing to a better quality of life when compared with those presented by the GC.
Key words: Aged. Women. Women's Health. Dancing. Muscle Stretching Exercises.

Comparative Study. Quality of Life. Meditation. Strengthening.

\section{INTRODUÇÃO}

O que se tem observado na população mundial é o envelhecimento populacional em países em desenvolvimento muito maior do que em países desenvolvidos. ${ }^{1} \mathrm{Na}$ atualidade, a população idosa no Brasil representa mais de $10 \%$ e, a partir de 2006, a expectativa do brasileiro chegou a 72,3 anos. ${ }^{2}$

A melhoria no acesso aos serviços básicos de saúde pública, a consolidação de um sistema universal e socializado de saúde pública no Brasil (o SUS), a maior eficiência das estratégias de vacinação em larga escala e de prevenção de doenças infecciosas, os avanços tecnológicos da medicina, o aumento do acesso à rede pública de saneamento, a melhoria dos níveis de escolaridade e o próprio enriquecimento global do país contribuíram para o aumento da expectativa de vida. ${ }^{3}$

Segmento heterogêneo da população, enquanto $80 \%$ das pessoas idosas estão bem de saúde, mantendo íntegras sua indepen- 
dência e autonomia, os outros $20 \%$ representam um grupo menos saudável, necessitando de cuidados mais intensos com equipes especializadas. ${ }^{4}$ As alternativas que melhor apresentam custo-benefício na compressão da morbidade são a promoção da saúde e a profilaxia primária e secundária de doenças. ${ }^{5}$

Mas, como se vê este idoso? Saber o que pensa e o que deseja para a própria vida é importante para serem definidos programas de atendimento que proporcionem lazer, entretenimento, saúde e qualidade de vida, mas sob o olhar de quem irá utilizá-lo: o idoso. ${ }^{6}$

A visão de qualidade de vida relacionada à autoestima e ao bem-estar pessoal envolve aspectos como a capacidade funcional, o nível socioeconômico, o estado emocional, a atividade intelectual, os valores culturais, éticos e a religiosidade e a própria saúde, o ambiente onde vive, as atividades e o estilo de vida cotidiano. ${ }^{7}$ Nesta perspectiva, existe a constatação que os estados físico, psicológico, social, cultural, mental e espiritual são fatores influenciadores da qualidade de vida do idoso, que não é um agente passivo, mas, dentro do contexto social, é ativo. ${ }^{8}$

Como decorrência desta constatação para a manutenção da saúde como um todo, e consequentemente, da qualidade de vida, a atividade física regular pode retardar os declínios relacionados ao envelhecimento, proporcionando maior longevidade, redução das taxas de morbidade e mortalidade, manutenção da independência e da autonomia, melhorando a autoimagem e a autoestima. ${ }^{5}$
Desta forma, o presente estudo objetiva avaliar os níveis de qualidade de vida de idosas ativas, participantes de um programa de dança, musculação e meditação, comparando ainda estas variáveis com as daquelas encontradas em idosas sedentárias.

\section{MATERIAIS E MÉTODOS}

\section{Amostra}

Para este estudo expostfacto, houve a participação de 50 idosas voluntárias, praticantes de atividades físicas (dança e musculação) e de meditação na casa do idoso do Programa de Saúde da Prefeitura Municipal de Belém do Pará. Também participaram da pesquisa 20 idosas sedentárias (não praticantes de atividades físicas regulares), voluntárias, que pertencem à comunidade ao redor da casa do idoso. Desta forma, a amostra foi dividida em quatro grupos: grupo de dança (GD, $n=20$; idade $=67 \pm 4$ anos; $\mathrm{IMC}=25,78 \pm 3,93)$, grupo de musculação (GF, $\mathrm{n}=15$; idade $=67 \pm 5$ anos; $\mathrm{IMC}=$ $27,12 \pm 4,66)$, grupo de meditação (GM, $\mathrm{n}=15$; idade $=68 \pm 4$ anos; $\mathrm{IMC}=27,17 \pm$ 2,46) e grupo controle (GC, $\mathrm{n}=20$; ida$\mathrm{de}=68 \pm 6$ anos; $\mathrm{IMC}=26,19 \pm 4,09)$.

Foram considerados critérios de inclusão as idosas que atendessem aos requisitos de saúde e ao consentimento livre e esclarecido referente aos objetivos deste estudo; como ativas, obtivessem $75 \%$ de presença nas atividades de musculação, dança ou meditação e que praticassem estas aulas há pelo menos seis meses; como sedentárias, 
não devessem estar fazendo atividades físicas há pelo menos três meses. ${ }^{9,10}$ Como critérios de exclusão, estavam os indivíduos do sexo masculino. Também foram impossibilitadas de participar da amostra as idosas que fizessem uso de medicamentos que pudessem causar distúrbios da atenção.

O presente estudo atendeu às normas para realização de pesquisas com seres humanos, conforme os procedimentos da Resolução no 196/96 do Conselho Nacional de Saúde, ${ }^{11}$ e foi aprovado pelo Comitê de Ética em Pesquisa da Universidade Castelo Branco/RJ. Todos os participantes assinaram o termo de consentimento livre e esclarecido referente à pesquisa.

\section{Procedimentos}

Para a avaliação da massa corporal e da estatura, foi utilizada uma balança mecânica com estadiômetro, precisão de 100 gramas e capacidade para 150 quilos (Filizola, Brasil), para então calcular-se o índice de massa corporal (IMC) ou índice de Quetelet.

Avaliação da Qualidade de vida: para esta variável, foi utilizado o questionário World Health Organization Quality of Life Group-oldWHOQOL-OLD, o qual é subdividido em seis domínios: Habilidades sensoriais (DOM1), Autonomia (DOM2), Atividades passadas presentes e futuras (DOM3), Participação social (DOM4), Morte e morrer (DOM5), Intimidade (DOM6). Estes domínios resultarão na análise da qualidade de vida geral - QVG. ${ }^{12}$
Grupo de Meditação: a meditação era realizada três vezes na semana, com duração de 50 minutos por sessão. Partindo da posição sentada, com olhos fechados, ao som de músicas em ritmo lento e volume suave, a sessão era dividida em três etapas: (a) controle respiratório, com respiração lenta e profunda, com o objetivo de reduzir a frequência respiratória e de ensinar a sequência correta de mobilização da caixa torácica. Esta respiração era seguida de concentração em diversas partes do corpo, da cabeça à pélvis; (b) repetição mental da palavra "paz", evitando qualquer outro tipo de pensamento. $\mathrm{O}$ ritmo respiratório era espontâneo. Esta fase foi baseada em técnica de resposta de relaxamento; e (c) esta etapa consistia da meditação em lembranças agradáveis e saudáveis.

Grupo de Dança: as aulas de dança de salão tinham 50 min de duração e ocorriam três vezes por semana. Os ritmos eram variados, a saber: foxtrot, valsa, rumba, swing, cha-cha e tango. A estrutura básica da aula era a mesma para todos os participantes, mas cada geronte possuía seu próprio desenvolvimento (progressão), dependendo de sua capacidade física, nível de energia, motivação e capacidade cognitiva. Todas as aulas eram precedidas de um período de aquecimento e flexibilidade e terminavam com relaxamento.

Grupo de Musculação: o GF realizava seu programa de treinamento $(2 \times 8-10$ repetições; $75-85 \%$ de $1-\mathrm{RM}$ ) com uma série do tipo alternada por segmento corporal dos seguintes exercícios: supino reto (SR), leg 
press $45^{\circ}\left(\operatorname{Leg} 45^{\circ}\right)$, puxada por trás no pulley alto (PT), flexão de pernas (FP), rosca bíceps com halteres $(\mathrm{RB})$, extensão de pernas (EP), rosca tríceps no pulley (RT) e abdominais (ABD). A frequência dos sujeitos ao treinamento foi de três vezes por semana em dias alternados e a sessão foi composta de um aquecimento, trabalho principal e relaxamento, perfazendo um tempo total de aproximadamente 45 minutos.

\section{Tratamento estatístico}

O tratamento estatístico foi composto por análise descritiva, através de medidas de localização (Média e Mediana) e de dispersão (Desvio-padrão - s, erro padrão da média - e). A normalidade da amostra foi testada através da análise inferencial de Shapiro-Wilk. ${ }^{13}$ A homogeneidade de variân- cia foi verificada com o teste de Levene. Para testar as diferenças entre os grupos, foi utilizado o Teste de Kruskal-Wallis ou de ANOVA one way, quando apropriado, seguido das comparações múltiplas através do intervalo de confiança (IC), ou do Post Hoc de Sheffe, respectivamente. Foi utilizado o pacote estatístico SPSS 14.0 e o programa Excell.

\section{RESULTADOS}

A tabela 1 traz os dados descritivos e a estatística inferencial de Shapiro Wilk da amostra. Nela pode-se observar que as variáveis dom1 (GD, GF e GC), dom2 (GD), dom3 (GM e GC), dom5 (GD, GF, GM e GC) e dom6 (GD) apresentaram distribuição heterogênea dos dados. ${ }^{13}$ 
Tabela 1 - Análise descritiva e inferencial de Shapiro Wilk da amostra. Belém, PA, 2007.

\begin{tabular}{|c|c|c|c|c|c|c|}
\hline & & $\mathrm{x}$ & e & Md & $s$ & SW \\
\hline \multirow{4}{*}{$\operatorname{dom} 1$} & GD & 18,50 & 0,46 & 19,00 & 2,04 & $\mathrm{p}<0,001$ \\
\hline & GF & 17,47 & 0,68 & 19,00 & 2,64 & 0,003 \\
\hline & GM & 17,13 & 0,65 & 17,00 & 2,50 & 0,064 \\
\hline & GC & 15,60 & 0,62 & 15,50 & 2,76 & 0,006 \\
\hline \multirow{4}{*}{$\operatorname{dom} 2$} & GD & 17,40 & 0,43 & 17,50 & 1,90 & 0,044 \\
\hline & GF & 15,33 & 0,63 & 15,00 & 2,44 & 0,233 \\
\hline & GM & 17,27 & 0,41 & 18,00 & 1,58 & 0,149 \\
\hline & GC & 12,50 & 0,39 & 12,00 & 1,76 & 0,321 \\
\hline \multirow{4}{*}{ dom 3} & GD & 17,00 & 0,46 & 17,00 & 2,08 & 0,270 \\
\hline & GF & 16,67 & 0,47 & 17,00 & 1,84 & 0,175 \\
\hline & GM & 17,73 & 0,33 & 18,00 & 1,28 & 0,026 \\
\hline & GC & 14,70 & 0,47 & 15,00 & 2,11 & 0,012 \\
\hline \multirow{4}{*}{$\operatorname{dom} 4$} & GD & 17,60 & 0,33 & 18,00 & 1,47 & 0,171 \\
\hline & GF & 17,33 & 0,52 & 17,00 & 2,02 & 0,190 \\
\hline & GM & 18,07 & 0,32 & 18,00 & 1,22 & 0,080 \\
\hline & GC & 14,90 & 0,61 & 15,00 & 2,71 & 0,058 \\
\hline \multirow{4}{*}{$\operatorname{dom} 5$} & GD & 17,20 & 0,65 & 18,00 & 2,91 & $\mathrm{p}<0,001$ \\
\hline & GF & 16,27 & 0,85 & 18,00 & 3,28 & 0,002 \\
\hline & GM & 17,20 & 0,39 & 18,00 & 1,52 & $\mathrm{p}<0,001$ \\
\hline & GC & 15,70 & 0,89 & 17,00 & 4,00 & 0,013 \\
\hline \multirow{4}{*}{$\operatorname{dom} 6$} & GD & 15,40 & 0,53 & 15,50 & 2,35 & 0,035 \\
\hline & GF & 13,53 & 1,00 & 15,00 & 3,87 & 0,174 \\
\hline & GM & 14,00 & 0,59 & 14,00 & 2,27 & 0,794 \\
\hline & GC & 14,35 & 0,85 & 15,00 & 3,80 & 0,139 \\
\hline \multirow{4}{*}{ QVG } & GD & 17,18 & 0,30 & 17,42 & 1,34 & 0,777 \\
\hline & GF & 16,10 & 0,42 & 16,50 & 1,61 & 0,087 \\
\hline & GM & 16,90 & 0,18 & 16,83 & 0,70 & 0,157 \\
\hline & GC & 14,62 & 0,38 & 14,67 & 1,71 & 0,419 \\
\hline
\end{tabular}

GD: grupo de dança; GF: GF: grupo de musculação; GM: grupo de meditação; GC: grupo controle; $x=$ média; e= erro padrão da média; $M d=$ mediana; $s=$ desvio padrão; $S W=$ estatística do teste Shapiro-Wilk; $Q V G=$ qualidade de vida geral; Dom1: Domínio do Funcionamento do Sensório; Dom2: Domínio da Autonomia; Dom3: Domínio das Atividades Passadas, Presentes e Futuras; Dom4: Domínio da Participação Social; Dom5: Domínio da Morte e Morrer; Dom6: Domínio da Intimidade. 
A homogeneidade de variância revelou uma distribuição heterogênea dos dados nos $\operatorname{dom} 4(p=0,042)$ e dom5 $(p=0,023)$. Os resultados encontrados indicam a conveniência de se utilizar a estatística inferencial com instrumentos não-paramétricos para os dados não-homogêneos e paramétricos para aqueles que se apresentaram normais. Desta forma, a ANOVA one way mostrou diferença significativa nas variáveis dom 1 $(p=0,005)$, dom $2(p<0,001)$, dom 3 $(p<0,001)$ e QVG $(p<0,001)$ e o teste de
Kruskal-Wallis no dom5 ( $\mathrm{p}<0,001)$. Nas comparações múltiplas, foram encontrados resultados satisfatórios nas variáveis dom 1 (GDxGC, p = 0,006), dom2 (GDxGF, $p=0,026 ; G D x G C, p<0,001 ; G F x G C$, $\mathrm{p}=0,001 ; \mathrm{GMxGC}, \mathrm{p}<0,001)$, dom3 (GDxGC, $p=0,004$; GFxGC, $p=0,033$; GMxGC, $p<0,001)$, QVG (GDxGC, $\mathrm{p}<0,001 ;$ GFxGC, $\mathrm{p}=0,033$; GMxGC, $\mathrm{p}<0,001$ ) e dom4 (GDxGC, $\mathrm{IC}=+0,421,+$ 41,479; GMxGC, IC = +4,456, +48,804) gráfico 1.

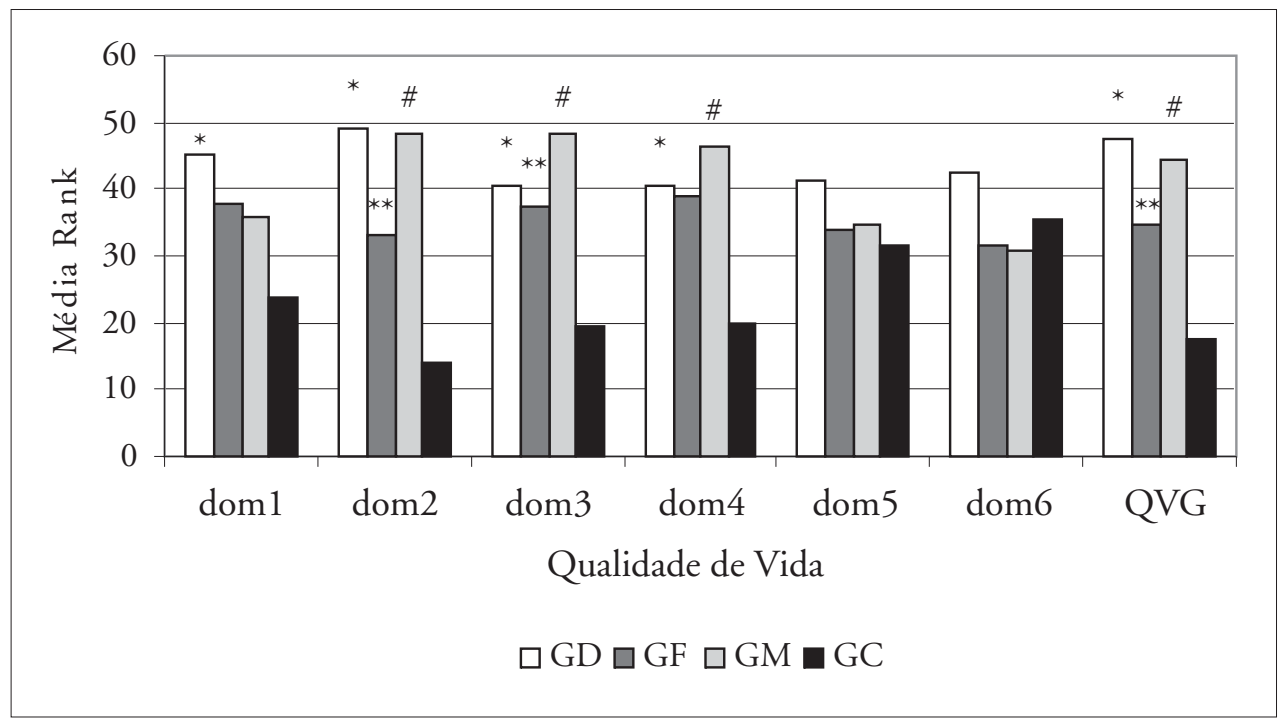

Gráfico 1: Avaliação das diferenças pela média do Rank nos domínios da qualidade de vida. Belém, PA, 2007.

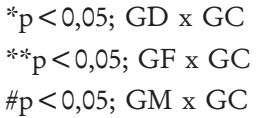

GD: grupo de dança; GF: GF: grupo de musculação; GM: grupo de meditação; GC: grupo controle; Dom1: Domínio do Funcionamento do Sensório; Dom2: Domínio da Autonomia; Dom3: Domínio das Atividades Passadas, Presentes e Futuras; Dom4: Domínio da Participação Social; Dom5: Domínio da Morte e Morrer; Dom6: Domínio da Intimidade. 


\section{DISCUSSÃO}

A avaliação dos efeitos dos programas de dança, musculação e meditação sobre os níveis de qualidade de vida das idosas revelou resultados significativos $(\mathrm{p}<0,05)$, quando comparados com o grupo de idosas que não estavam inseridas em nenhum tipo de atividade física regular. Em uma revisão de literatura, Stella et al. ${ }^{14}$ observaram que a depressão representa enfermidade mental frequente no idoso, comprometendo de forma significativa sua qualidade de vida. Entretanto, quando o idoso está engajado em um programa de atividade física, regular e bem planejado, isso contribui para a minimização do sofrimento psíquico do idoso deprimido, além de oferecer oportunidade de envolvimento psicossocial, elevação da autoestima e implementação das funções cognitivas, fatores estes muito importantes no contexto dessa população.

Em estudo experimental que objetivou comparar o nível de qualidade de vida entre idosos que praticassem uma atividade física e aqueles que não praticassem, foi observado que o grupo controle ( $\mathrm{n}=42$ idosos), sedentário, teve pontuação significativamente menor em todos os domínios do teste (Short-form 36 -SF-36), quando comparada ao do grupo experimental $(n=46$ idosos), que participou da atividade física. ${ }^{15}$ Estes resultados dão sustentação àqueles revelados na presente pesquisa, pois o gráfico 1 mostra que os grupos que participavam de alguma atividade obtiveram resultados satisfatórios em relação ao GC nos dom1 (GD), dom2 (GD, GF e GM), dom3 (GD, GF e GM), dom4 (GD e GM) e QVG (GD e GM).

Em estudo realizado por Vale, ${ }^{16}$ foi observado que os níveis de qualidade de vida de um grupo praticante de treinamento de força (GFOR) por 16 semanas melhoraram no pós-teste, principalmente o dom3 (nível de independência) e o dom4 (relações sociais) do questionário WHOQOL-100. Isto sugere que o GFOR, embora tenha conseguido perceber modificações positivas nestes domínios, não atingiu significativamente os outros domínios, embora o grupo tenha tido percepção na melhora de sua qualidade de vida. Estes dados corroboram os achados da presente pesquisa, pois o gráfico 1 revela melhora significativa em alguns domínios do WHOQOL-OLD (dom1, dom2, dom3, dom4 e QVG), embora dom5 e dom6 não tenham apresentado alterações intergrupos. ${ }^{12}$

Embora a meditação não seja considerada uma atividade física em sua essência, os dados do vigente estudo revelam, no gráfico 1, que as idosas praticantes de meditação (GM) obtiveram melhores níveis de qualidade de vida quando comparadas às do GC. Estes achados possuem a mesma tendência daqueles encontrados em estudo prospectivo com idosos portadores de insuficiência cardíaca congestiva. ${ }^{17}$ Através do questionário Minnesota Living with Heart Failure Questionnaire (MLWHFQ), foi observada melhora significativa $(p=0,02)$ na qualidade de vida do grupo experimental, que realizou a prática da meditação $(n=10)$, quando compa- 
rado com o grupo controle $(\mathrm{n}=9) \cdot{ }^{17}$ Estes dados ainda são sustentados pelo trabalho de Jayadevappa et al., ${ }^{18}$ os quais observaram, numa amostra com indivíduos com idade $>55$ anos, que a prática da meditação (grupo experimental, $n=13$ ) resultou em melhora significativa $(\mathrm{p}<0,05)$ da qualidade de vida (SF-36), quando comparada ao grupo de indivíduos que somente participavam de uma aula de "educação de saúde" (grupo controle, $n=10)$. Adicionalmente, foi relatada melhora significativa da capacidade funcional $(p=0,034)$ e da depressão (Epidemiologic Studies Depression Scale - CES-D - $\mathrm{p}=0,03)$.

Os dados expostos no gráfico 1 revelam que o GF apresentou níveis de qualidade de vida significativamente maiores $(p<0,05)$, quando comparados aos do GC. Esses resultados corroboram aqueles encontrados numa pesquisa realizada por Carta et al. ${ }^{19} \mathrm{Em}$ amostra com idosas portadoras de depressão, os autores observaram que no grupo experimental, que realizou um trabalho de força (musculação), houve melhora significativa $(p<0,05)$ na qualidade de vida, segundo o questionário WHOQOLbref. Entretanto, o grupo controle, que permaneceu sedentário e apenas com uso de medicação antidepressiva, não obteve diferença no nível de qualidade de vida.

A presente pesquisa revelou que a prática de atividade física (dança e musculação) e de meditação se refletiu em resultados estatisticamente satisfatórios $(\mathrm{p}<0,05)$ na qualidade de vida, quando comparados ao GC. Antunes et al. ${ }^{20}$ avaliaram os efeitos do treinamento aeróbico no nível de qualidade de vida de idosos saudáveis (idade $=$ $66,97 \pm 4,80$ anos). Para tal, dividiu sua amostra em grupo experimental (GE, $\mathrm{n}=23$ ) e grupo controle ( $\mathrm{GC}, \mathrm{n}=23$ ). $\mathrm{O}$ questionário utilizado foi o SF-36, que revelou melhora significativa $(p<0,05)$ na qualidade de vida em seus resultados intra e intergrupos, favoráveis ao GE.

Contrapondo os dados do vigente estudo, Paw et al..$^{21}$ observaram que, em idosos asilados, o treinamento isolado de força e de recreação não repercutiram em melhora significativa da qualidade de vida (Dementia Quality of Life questionnaire - DQoL). O resultado significativo $(\mathrm{p}<0,05)$ só ocorreu no grupo que fez o trabalho conjugado (força + recreação), quando comparado ao grupo controle.

\section{CONCLUSÃO}

Após a exposição dos dados, inferiu-se que os dois programas de atividade física (dança e musculação) e a prática da meditação apresentaram resultados satisfatórios na amostra analisada, , contribuindo para um melhor nível de qualidade de vida quando comparados com aqueles apresentados pelo GC.

A avaliação do WHOQOL-OLD, referente aos efeitos dos programas de atividade física sobre a qualidade de vida, não deixa dúvida de que principalmente a dança, mas também a musculação e a medição, atuaram elevando os níveis de qualidade de vida nas idosas praticantes destas atividades. 


\section{REFERÊNCIAS}

1. Pereira FF, Monteiro N, Vale RGS, Gomes ALM, Novaes JS, Júnior AGF, et al. Efecto del entrenamiento de fuerza sobre la autonomía funcional en mujeres mayores sanas. Revista Española de Geriatria e Gerontologia 2007; 42(6): 319-24.

2. IBGE. Base de Dados. 2004 [cited 2006 set. 10]; Available from: URL: http:// www.ibge.gov.br

3. Dias Jr CS, Costa CS, Lacerda MA. O envelhecimento da população brasileira: uma análise de conteúdo das páginas da REBEP. Revista brasileira de geriatria e gerontologia 2006; 9(2).

4. Veras RP, Caldas CP, Coelho FD, Sanches MA. Promovendo a Saúde e Prevenindo a Dependência: identificando indicadores de fragilidade em idosos independentes. Revista brasileira de geriatria e gerontologia 2007; 10(3).

5. Cardoso AS, Mazo GZ, Salin MS, Santos CAX. Percepção subjetiva de saúde e nível de atividade física de idosos. Revista brasileira de geriatria e gerontologia 2008; 11(1).

6. Jardim VCFS, Medeiros BF, Brito AM. Um olhar sobre o processo do envelhecimento: a percepção de idosos sobre a velhice. Revista brasileira de geriatria e gerontologia 2006; 9(2).

7. Vecchia RD, Ruiz T, Bocchi SCM, Corrente JE. Qualidade de vida na terceira idade: um conceito subjetivo. Revista brasileira de epidemiologia 2005; 8(3): 246-52.

8. Doimo LA, Derntl A. Uso do tempo no cotidiano de idosos: um método indicador do estilo e modo de vida na velhice. Revista brasileira de geriatria e gerontologia 2006; 9(1).
9. Kraemer WJ, Koziris LP, Ratamess NA, Hakkinen K, Triplett-Mcbride NT, Fry AC, et al. Detraining produces minimal changes in rhysical performance and hormonal variables in recreationally strength-trained men. J Strength Cond Res 2002; 16(3): 373-82.

10. Raso V, Matsudo SMM, Matsudo VKR. A força muscular de mulheres idosas decresce principalmente após oito semanas de interrupção de um programa de exercícios com pesos livres. Revista Brasileira de Medicina do Esporte 2001; 7(6): 177-86.

11. Conselho Nacional de Saúde. Resolução ${ }^{\circ}$ 196, de 10 de outubro de 1996. Brasília (DF): Conselho Nacional de Saúde; 1996.

12. Fleck M, Chachamovich E, Trentini C. Desenvolvimento e validação da versão em Português do módulo WHOQOL-OLD. Rev Saúde Pública 2006; 40(5): 785-91.

13. Thomas JR, Nelson JK, Silverman S. Métodos de pesquisa em atividade física. 5 ed. Porto Alegre: Artmed; 2005.

14. Stella F, Gobbi S, Corazza DI, Costa JLR. Depressão no idoso: diagnóstico, tratamento e benefícios da atividade física. Motriz 2002; 8(3): 91-8.

15. Mota J, Ribeiro JL, Carvalho J, Matos MG. Atividade física e qualidade de vida associada à saúde em idosos participantes e não participantes em programas regulares de atividade física. Revista brasileira de educação fisica e esporte 2006; 20(3): 219-25.

16. Vale RGS. Efeitos do treinamento de força e de flexibilidade sobre a autonomia e a qualidade de vida de mulheres senescentes. Rio de Janeiro: Universidade Castelo Branco; 2004.

17. CuriatiJA, Bocchi E, Freire JO, Arantes AC, Braga M, Garcia Y, et al. Meditation 
reduces sympathetic activation and improves the quality of life in elderly patients with optimally treated heart failure: a prospective randomized study. J Altern Complement Med 2005; 11(3): 465-72.

18. Jayadevappa R, Johnson JC, Bloom BS, Nidich S, Desai S, Chhatre S, et al. Effectiveness of Transcendental Meditation on Functional Capacity and Quality of Life of African Americans with Congestive Heart Failure: A Randomized Control Study. Ethn Dis 2007; 17(1): 72-7.

19. Carta MG, Hardoy MC, Pilu A, Sorba M, Floris AL, Mannu FA, et al. Improving physical quality of life with group physical activity in the adjunctive treatment of major depressive disorder. Clin Pract Epidemol Ment Health 2008; 4(1): 1-6.

20. Antunes HKM, Stella SG, Santos RF, Bueno OFA, Mello MTD. Depression, anxiety and quality of life scores in seniors after an endurance exercise program. Rev Bras Psiquiatr 2005; 27(4): 266-71.

21. Paw MJCA, Poppel MNV, Twisk JW, Mechelen WV. Effects of resistance and all-round, functional training on quality of life, vitality and depression of older adults living in long-term care facilities: a 'randomized' controlled trial. BMC Geriatr 2004; 4(5): 1-9. 


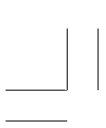

